\title{
Targeted Approach for Prostate Cancer Treatment: Synthesis and Characterization of Docetaxel-Loaded Perfluorocarbon Nanodroplets
}

\author{
Gupta R, Cvetkovic D, Ma CM and Chen $\mathrm{L}^{*}$ \\ Department of Radiation Oncology, Fox Chase Cancer Center, Philadelphia, PA, USA
}

*Corresponding author: Chen L, Department of Radiation Oncology, Fox Chase Cancer Center, Philadelphia, PA, USA- 19111, E-mail: lili.chen@fccc.edu

Citation: Gupta R, Cvetkovic D, Ma CM, Chen L (2014) Targeted Approach for Prostate Cancer Treatment: Synthesis and Characterization of Docetaxel-Loaded Perfluorocarbon Nanodroplets. J Cancer Sci Clin Oncol 1(1): 102. doi: 10.15744/2394-6520.1.102

Received Date: September 12, 2014 Accepted Date: December 02, 2014 Published Date: December 04, 2014

\begin{abstract}
The purpose of this study is to synthesize and characterize nanodroplets, loaded with docetaxel for treatment of prostate cancer under MR-guided focused ultrasound. Water insoluble docetaxel encapsulated in nanodroplets is expected to be delivered into tumors with greater efficiency while minimizing drug related systemic toxicities when used in combination with focused ultrasound. The sequence of our studies toward development and characterization of docetaxel-loaded nanodroplets is as follows. First, we developed methods for synthesis of ultrasound-responsive, docetaxel-loaded nanodroplets (Doc-nd) by a solid dispersion technique. Secondly, we characterized Doc-nd by its morphology and size distributions using a dynamic light scattering (DLS) method. To check the consistency of the data obtained from DLS, size distribution was also studied using an independent technique by analyzing bright field microscopy images of Doc-nd in software ImageJ. Thirdly, we studied the encapsulation efficiency and the release kinetics of docetaxel from Doc-nd in phosphate buffer saline. Finally, we performed in vitro cytotoxicity studies using a human prostate cancer cell line (LNCaP). Our data showed that peak sizes of spherical Doc-nd were $222 \pm 15 \mathrm{~nm}$. These sizes favor passive accumulation into most tumors thus potentially increasing localized docetaxel concentration. A high encapsulation efficiency of $93.70 \%$ was obtained for Doc-nD. The release kinetics studies showed that docetaxel was released from Doc-nd in a three- staged release pattern with an initial release of $30 \%$ in one hour followed by a $50 \%$ release until $12 \mathrm{~h}$ and an $85 \%$ release after three days. In vitro cytotoxicity studies using LNCaP cells indicated a time-dependent cytotoxic profile and verified encapsulation of docetaxel in the nanodroplets. Our study suggested that Doc-nd may have the potential for treating prostate cancer with an improved therapeutic ratio when combined with MR-guided focused ultrasound.
\end{abstract}

Keywords: Docetaxel; Perfluorocarbon Nanodroplets; Ultrasound; Prostate Cancer

\section{Introduction}

Treating prostate cancer remains a clinical challenge because of its heterogeneity and specific nature of individual cancers. Current standard leading therapies for local prostate cancer include radical prostatectomy, external beam radiation therapy (RT) and/or brachytherapy. For locally advanced stages or disseminated prostate cancer, additional therapies like androgen deprivation therapy (ADT) or chemotherapy are given. For treating local prostate cancer, apart from prostatectomy, RT is one of the most effective treatment modalities. Several radiation treatment strategies (eg. dose escalation) for treating prostate cancer have been used over the years but the optimal treatment choice lacks general consensus [1]. Biochemical failure or recurrence (local persistence of the disease and/or metastasis) occurs in about 35\% of patients within 10 years of receiving treatment [2,3]. ADT is one of the common standards of care for such patients but there are many controversies in managing biochemical failure using ADT [2]. Chemotherapy has shown to prolong survival in metastatic/advanced prostate cancer patients [4,5]. Docetaxel, as an anti-mitotic chemotherapy agent, has routinely been used clinically for treating prostate cancer patients however as with all chemotherapy, high normal tissue toxicity and low bio-availability have been documented for this type of treatment therapy [6-8].

Recent publications report new treatment modalities like High-intensity focused ultrasound (HIFU) for prostate cancer which are being evaluated with the aim of decreasing complication rates associated with the standard treatment options, as well as developing effective treatment $[9,10]$. HIFU has recently been promoted as a non-invasive treatment option for prostate cancer. HIFU is not yet approved for use in prostate cancer treatment in the Unites States by the FDA, but it is used clinically worldwide in countries like Canada, Japan, and Europe etc.

Our goal to initiate the present study is to develop a novel approach for treating prostate cancer with improved efficiency while reducing normal tissue toxicities. In this treatment approach, docetaxel is delivered into the tumor via encapsulation inside an ultrasound-sensitive, drug delivery system. Once the system reaches tumor, MR-guided HIFU (MRg-HIFU) is applied to the tumor to help activate the release of docetaxel from nanodroplets inside the tumor, thus increasing the bioavailability of the drug. 
Drug-delivery systems for chemotherapeutics have the potential to provide a method to maximize the localization of the drug into tumor tissues while minimizing normal tissue toxicity [11-14]. Previous studies have shown that tumor tissues in general are characterized by poorly organized vascular architecture, irregular blood flow and reduced lymphatic drainage. These properties are exploited by drug delivery systems of appropriate sizes to accumulate in tumor tissues via enhanced permeability and retention (EPR) effects [15-17]. Blood vessels in normal tissues have tight inter-endothelial junctions (usually having a diameter of less than $7 \mathrm{~nm}$ ), which do not allow extravasation of these drug carriers into normal tissues thus reducing normal tissue toxicity. The tumor endothelial pore cutoff size for most of the tumor types ranges from $100 \mathrm{~nm}$ to $780 \mathrm{~nm}$ [18] which allows extravasation of these drug-loaded, drug-delivery vehicles into the tumor, thus increasing bioavailability of the drug inside the tumor. Hence, in order to reduce systemic toxicity of docetaxel and improve its bioavailability to the prostate tumor, we have developed techniques to synthesize and characterize ultrasound-responsive, docetaxel-loaded nanodroplets (Doc-nd). We have also evaluated the cytotoxic effect of Doc-nd on prostate tumors in vitro using a human prostate cancer cell line (LNCaP). These nanodroplets exhibit ultrasound-responsive properties due to the presence of perfluorocarbon-15-crown-5-ether (PFCE) in their core [13]. PFCE imparts cavitating properties to the nanodroplets. When nanodroplets accumulate in the tumor due to EPR effect and tumor-directed ultrasound is applied, these drug-loaded nanodroplets transform into oscillating, cavitating bubbles resulting in a very high, efficient localized release of the drug into the tumor [13] therefore increasing its bioavailability.

Our long-term goal is to test the hypothesis that MR-guided HIFU (MRg-HIFU) can be used as a tool to activate docetaxel-loaded nanodroplets (Doc-nd) localized in the target prostate tumor (in vivo) hence killing cancer cells effectively while significantly reducing normal tissue toxicities leading to a much improved therapeutic ratio. The present study is the first step in testing the above hypothesis and reports the preparation of the novel drug-delivery formulation (docetaxel-loaded, ultrasound-sensitive nanodroplets) and its characterization with respect to their sizes, morphology, encapsulation efficiency, release profile and in vitro cytotoxic properties against human prostate cancer cells.

\section{Materials and Methods}

\section{Materials}

Block copolymer, poly (ethylene oxide)-co-poly (D, L-lactide) (PEG-PDLA) (2000Da- 2000Da) was purchased from Akina, Inc. (West Lafayette, IN). Docetaxel (Doc) (Molecular mass $807.879 \mathrm{~g} / \mathrm{mol}$ ) of more than $97.0 \%$ purity was purchased from SigmaAldrich (St. Louis, MO) and used without further purification. Perfluorocarbon-15-crown-5-ether (PFCE) was purchased from Oakwood Products, Inc. (West Columbia, SC). The solvent tetrahyrdrofuran (analytical grade) was purchased from Sigma-Aldrich (St. Louis, MO).

\section{Synthesis of docetaxel-loaded PFCE nanodroplets (Doc-nd)}

Doc-nd was prepared by the solid dispersion technique. This technique has been used by other research groups for synthesizing paclitaxel formulations [11-13,19]. Briefly, $1 \mathrm{mg}(0.1 \%)$ of Doc (obtained from $5 \mathrm{mg} / 100 \mu \mathrm{l}$ of stock in dimethyl sulfoxide) and 20mg (2\%) of amphiphilic block co-polymer PEG-PDLA were co-dissolved in $1 \mathrm{ml}$ of tetrahydrofuran (THF). The THF was then evaporated under gentle nitrogen stream at $60{ }^{\circ} \mathrm{C}$ or evacuated in a vacuum at room temperature. The residual gel matrix consequently obtained was dissolved in $1 \mathrm{ml}$ of phosphate buffer saline (PBS, pH 7.4) to form polymeric micelles. Empty micelles (without drug) were formed in the same way. The micellar solutions obtained were sterilized by filtration through $200 \mathrm{~nm}$ filters. Subsequently, an aliquot of sterilized PFCE $(20 \mu \mathrm{l}-2 \%)$ was introduced into this micellar solution and the mixture was emulsified on ice externally using a $20 \mathrm{kHz}$ ultrasonic processor (VCX500, Sonics and Materials, Inc; CT, USA) at 35\% amplitude. Thus empty nanodroplets (2\%PFCE/2\%PEG-PDLA) and Doc-nd (0.1\%DTX/2\%PFCE/2\%PEG-PDLA) of the given composition were synthesized.

The concentration of Doc $(0.1 \%)$ used for encapsulation in the nanodroplets was in accordance with our previous in vivo (5mg per kg) Doc related studies [20-21]. Amphiphilic block copolymers, which are composed of both hydrophobic and hydrophilic segments, have the capacity to self-assemble into nanoscale spherical structures with a hydrophilic outer shell and a hydrophobic inner core [22-24]. Poor water soluble drugs can be incorporated into the hydrophobic part while the hydrophilic outer shell exists as a stabilizer for the system. These systems are often termed as micelles [11-13]. PEG is used as the hydrophilic outer shell due to its biocompatibility, low toxicity, negligible antigenicity and immunogenicity $[13,14]$. To become ultrasound responsive (i.e. to be able to convert into bubbles under the action of ultrasound), perfluorocarbons are added to these micelles and sonicated to form nanodroplets [11-13,25].

\section{Characterization of Doc-loaded PFCE nanodroplets}

Size distribution and surface morphology: The mean diameter and size distributions of the nanodroplets were measured at 25 ${ }^{\circ} \mathrm{C}$ by dynamic light scattering (DLS) at a scattering angle of $90^{\circ}$ using a DynaPro Plate Reader (Wyatt Technology, Santa Barbara, CA) equipped with a temperature controller. Morphology of the nanodroplets was visualized using a 60X objective of the Nikon Eclipse inverted TE 2000 optical microscope (Tokyo, Japan) and images were captured using an attached CCD camera (Cascade 650, Roper Scientific, NJ). 
The particle diameter statistics were also obtained by analyzing bright field images of nanodroplets using ImageJ (Image processing and analysis in Java). ImageJ is a public domain, Java-based image processing program developed at the National Institutes of Health (http://imagej.nih.gov/ij/). A measurement scale was set by drawing a line between two points of known distance using the Set Scale. The option 'global' was checked to apply this scale to all image frames. Feret's diameter was chosen as a measuring parameter in Set Measurements. Five independent fields from the acquired images were selected randomly and analyzed individually. For each visual droplet on the image, a line was drawn manually from one point to another point such that the maximum diameter of the droplet was covered. From the "Measure" option, the diameter of the droplet was obtained.

Drug encapsulation efficiency: The encapsulation efficiency was calculated by an indirect method using the following formula: Encapsulation efficiency $(\%)=$ [Total drug added $(\mu \mathrm{g})$ - free drug $(\mu \mathrm{g}) /$ Total drug added $(\mu \mathrm{g})]$ x 100 [26]. For this, $1 \mathrm{ml}$ of Docnd $(0.1 \% \mathrm{DTX} / 2 \% \mathrm{PFCE} / 2 \%$ PEG-PDLA) was centrifuged (Eppendorf Centrifuge 5415D, Hauppauge, NY) at $13200 \mathrm{rpm}$ for 10 min at $4{ }^{\circ} \mathrm{C}$ [27]. The supernatant was removed and analyzed for free Doc concentration by measuring UV absorbance at 227nm using a NanoVue Spectrophotometer (GE Healthcare, Pittsburgh, PA) [14]. To determine the exact drug content, an absorbance calibration curve of known Doc concentrations $(10-1000 \mu \mathrm{g} / \mathrm{ml}$ in DMSO) was obtained at 227nm. A graph was plotted between the known concentrations and absorbance. From this graph, a linear equation was obtained which was used to determine the exact concentration of free Doc in the supernatant.

In vitro release kinetics: The release of Doc from Doc-nd was assessed in PBS ( $\mathrm{pH} 7.4$ ) [27,28]. One ml of Doc-nd (0.1\%DTX/2\%PFCE/2\%PEG-PDLA) was diluted with $9 \mathrm{ml}$ of PBS to get $10 \mathrm{ml}$ of the suspension in a screw-capped tube and placed in an orbital shaker (Bellco Biotechnology, Vineland, NJ), which was maintained at $37^{\circ} \mathrm{C}$ and shaken horizontally at $120 \mathrm{rpm}$ [27]. At specific time intervals $(5 \mathrm{~min}, 30 \mathrm{~min}, 1 \mathrm{~h}, 3 \mathrm{~h}, 6 \mathrm{~h}, 12 \mathrm{~h}$ and 3 days), the tube was taken out of the shaker and centrifuged at $13200 \mathrm{rpm}$ for $10 \mathrm{~min}$ at $4{ }^{\circ} \mathrm{C}$. The precipitated nanodroplets were re-suspended in $10 \mathrm{ml}$ of fresh buffer before being returned to the shaker until the next specific time interval. This was done to keep the incubation medium at a constant volume. The supernatant was analyzed for free Doc concentration by measuring UV absorbance at wavelength $227 \mathrm{~nm}$. It should be indicated that the concentration of released Doc was corrected for the dilution due to adding PBS.

In vitro cytotoxicity/proliferation study: Cell cytotoxicity of Doc-nd (0.1\%DTX/2\%PFCE/2\%PEG-PDLA), empty nanodroplets (empty-nd) (2\%PFCE/2\%PEG-PDLA) and free Doc were assessed by cell proliferation reagent WST-1 (Roche Applied Science, Indianapolis, IN). Human prostate cancer LNCaP cells were obtained from the American Type Culture Collection and cultured in Dulbecco's modified Eagle's medium, (DMEM)-F12 medium, containing 10\% fetal bovine serum (FBS), 1\% L-glutamine and $1 \%$ penicillin-streptomycin. LNCaP cells $\left(5 \times 10^{3} /\right.$ well) were seeded in 96 -well plates. After $24 \mathrm{~h}$, adhered cells were exposed to free Doc (the same concentration as used for encapsulation in the nanodroplets) and Doc-nd (in separate experiments) at $37^{\circ} \mathrm{C}$ and at $5 \% \mathrm{CO}_{2}$ for different time intervals (2min, $5 \mathrm{~min}, 15 \mathrm{~min}, 30 \mathrm{~min}, 1 \mathrm{~h}, 3 \mathrm{~h}, 6 \mathrm{~h}, 12 \mathrm{~h}$, and 3 days). After specific time points, cells were washed with PBS and fresh media with serum was added. Cells were then allowed to grow for 3 days at $37^{\circ} \mathrm{C}$ and an assay was performed using WST-1. It was read at wavelength 450nm on the Envision 2102 Multilabel Reader (Perkin Elmer, Waltham, MA). The data were expressed as a percentage of untreated/control cells.

\section{Data Analysis}

All experiments were performed in independent triplicates. Each result is presented as mean \pm SD with $n=3$.

\section{Results and Discussion}

\section{Size distribution by dynamic light scattering (DLS)}

The particle size distribution for both Doc-nd (0.1\%DTX/2\%PFCE/2\%PEG-PDLA) and E-nd (2\%PFCE/2\%PEG-PDLA) when measured by the DLS method is presented in Figure $1 \mathrm{~B}$ and $1 \mathrm{~A}$ respectively. The peak size of $222 \pm 15 \mathrm{~nm}$ for Doc-nd was found to be greater than the peak size for E-nd $(184 \pm 25 \mathrm{~nm})$ indicating an increase in size after encapsulation of docetaxel in the nanodroplets. The size distribution obtained for Doc-nd would be optimal for favorable accumulation of these into the tumor via the EPR effect, as most tumors have pore cutoff sizes ranging from $150 \mathrm{~nm}$ to a micron $[13,16,17]$. Sizes larger than mentioned above may cause clogging of blood vessels when used for intravenous injections for in vivo experiments. Empty PEG-PDLA micellar solution was found to be $89 \pm 20 \mathrm{~nm}$ (peak size) and Doc loaded PEG-PDLA micelles were found to be $110 \pm 15 \mathrm{~nm}$ (data not shown). This increase in micellar size again indicates encapsulation of docetaxel. To this micellar solution, PFCE was added to form nanodroplets. Typically, with PFCE and PEG-PDLA micellar solution, bimodal distribution of nanodroplets is observed indicating a mixture of micelles and nanodroplets [13]. On the basis of size of empty and docetaxel-loaded PEG-PDLA micelles, the size distribution of nanodroplets suggests that smaller sized particles in these represent micelles. Thus, the nanodroplets obtained after emulsification of the micellar solution with PFCE contain both micelles and nanodroplets. We reported earlier that the micelle/droplet ratio decreases with an increasing PFCE concentration; micelles disappeared at a PFCE concentration of $5 \%$ indicating transfer of all block copolymer onto droplet surfaces [13]. In the present work, we have used 2\% PFCE and 2\% PEG-PDLA to form nanodroplets based on our previous studies [13]. This composition was used to prepare nanodroplets which favor the EPR effect thus allowing efficient extravasation into the tumor tissues. Further optimization with respect to a different concentration of PFCE and PEG-PDLA (with and without Doc) was not the scope of the present work and will be done in the future if needed based on the in vivo studies. 

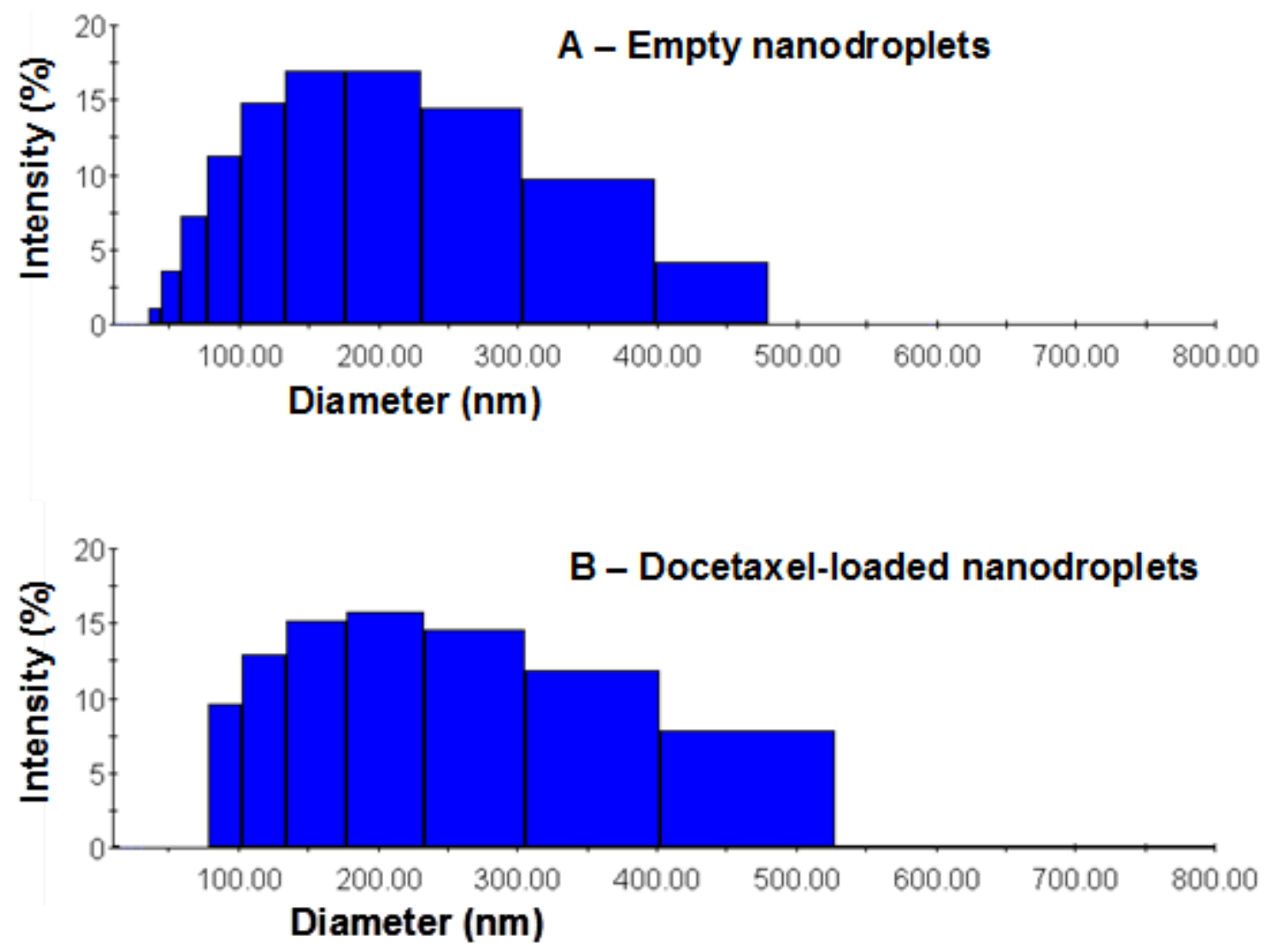

Figure 1: Size distribution by Dynamic light scattering method. (A) Empty nanodroplets (2\%PFCE/2\%PEG-PDLA), peak sizes - $184.03 \pm 24.50$ $\mathrm{nm}$ and (B) Docetaxel-loaded nanodroplets (0.1\%DTX/2\%PFCE/2\%PEG-PDLA), peak sizes - 222.01 $14.67 \mathrm{~nm}$

\section{Surface morphology}

The high magnification optical microscopy suggested that all nanodroplets (both Doc-nd and E-nd) had a fine spherical shape. Bright field microscopy image at 60X for Doc-nd is shown in Figure 2A. The image indicated a heterogeneous mixture of nanodroplets i.e. droplets of varying sizes. Some droplets in the micron range were also observed in these images which were not seen in dynamic light scattering data. Perhaps these droplets avoided measurement by DLS due to precipitation to the bottom of the cuvette during the measurement process. Each nanodroplet structure corresponds to the schematic diagram as shown in Figure 2B. The central portion consisted of a PFCE loaded liquid core surrounded by a docetaxel-loaded hydrophobic part of the block copolymer and stabilizing outer-most hydrophilic portion of the polymer.

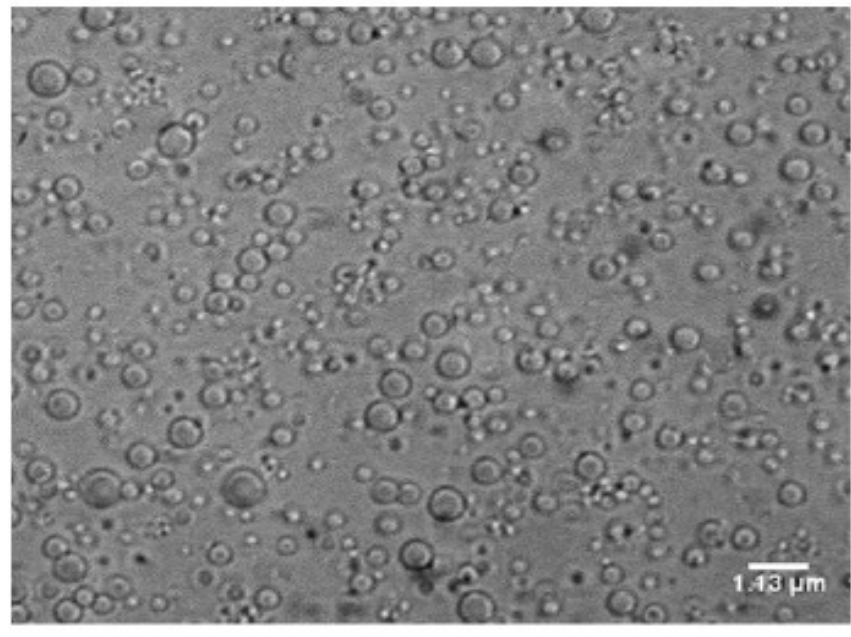

A

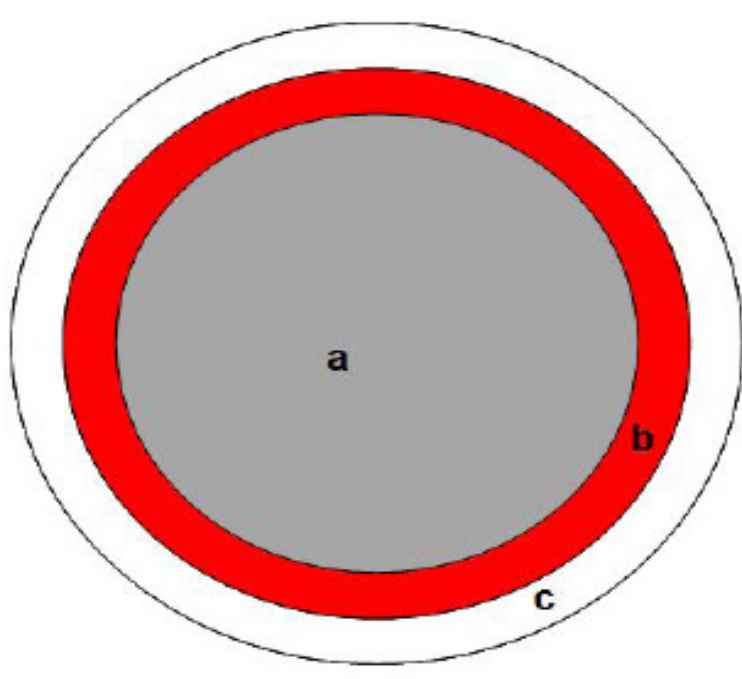

B

Figure 2: Docetaxel-loaded nanodroplets. (A) Bright field Image of docetaxel-loaded nanodroplets (0.1\%DTX/2\%PFCE/2\%PEG-PDLA) obtained using 60X objective of Nikon eclipse inverted TE 2000 microscope. (B) Schematic diagram of a nanodroplet showing internal liquid perfluorocarbon core as "a", hydrophobic shell of a block copolymer where hydrophobic drug (docetaxel) is encapsulated as "b" (shown in red color), and outer hydrophilic shell of the block copolymer as "c" 


\section{Size distribution by image analysis}

In order to check the consistency of the size measurements with independent techniques, images as obtained after visualizing nanodroplets using high magnification optical microscope were also analyzed for size/diameter using ImageJ. One-hundred nanodroplets from each of the five randomly selected fields were analyzed individually (for both Doc-nd and E-nd). The size distributions (diameter) were grouped in different size ranges and frequencies (number of droplets in each size range) were obtained (Table 1). The image analysis data was found to be comparable with DLS data (Figure 1). The highest frequency/number of droplets were found to be in the diameter range of 200-250 nm and 150-200 nm for Doc-nd and E-nd respectively (Table 1circled with red). These are similar to the peak size of $222 \pm 15 \mathrm{~nm}$ for Doc-nd and peak size of $184 \pm 25 \mathrm{~nm}$ for E-nd as measured using DLS. Image analysis distribution (Table 1) also showed some droplets in a range of $>550 \mathrm{~nm}$ for both E-nd and Doc-nd. These large sized nanodroplets are not seen in the DLS data (Figure 1). Thus, measuring size distribution using Image analysis is more advantageous than measuring by using only DLS since it provides more complete information about heterogeneity of the nanodroplets.

\begin{tabular}{|c|c|c|}
\hline $\begin{array}{l}\text { Range of nanodroplet sizes } \\
\text { with respect to diameter(nm) }\end{array}$ & $\begin{array}{l}\text { Frequency for Docetaxel } \\
\text { loaded nanodroplet } \\
(0.1 \% \text { DXT/2\%PFCE/2\%PEG-PDLA) }\end{array}$ & $\begin{array}{l}\text { Frequency for Empty } \\
\text { nanodroplets } \\
\text { (2\%PFCE/2\%PEG-PDLA) }\end{array}$ \\
\hline $25-50$ & 0 & 5 \\
\hline $50-100$ & 12 & 15 \\
\hline $100-150$ & 25 & 31 \\
\hline $150-200$ & 67 & 119 \\
\hline $200-250$ & 121 & 69 \\
\hline $250-300$ & 63 & 60 \\
\hline $300-350$ & 52 & 55 \\
\hline $350-400$ & 28 & 27 \\
\hline $400-450$ & 26 & 24 \\
\hline $450-500$ & 21 & 19 \\
\hline $500-550$ & 21 & 21 \\
\hline $550-600$ & 12 & 13 \\
\hline $600-650$ & 11 & 11 \\
\hline $650-700$ & 15 & 14 \\
\hline $700-750$ & 10 & 6 \\
\hline $750-800$ & 9 & 8 \\
\hline $800-850$ & 7 & 3 \\
\hline $850-900$ & 0 & 0 \\
\hline
\end{tabular}

Table 1: Size distribution analysis for images using software package ImageJ. The data shows the size distribution statistics with respect to diameter for empty (2\%PFCE/2\%PEG-PDLA) and docetaxel-loaded (0.1\%DTX/2\%PFCE/2\%PEGPDLA) nanodroplets. Details are mentioned in previous section.

\section{Docetaxel encapsulation efficiency}

Encapsulation efficiency was determined to obtain the total amount of the drug (docetaxel) present in the nanodroplets. It was detected by measuring the non-incorporated drug present in supernatant after separation of nanodroplets by centrifugation. A calibration curve of known docetaxel concentrations $(10-1000 \mu \mathrm{g} / \mathrm{ml})$ was plotted against absorbance at $227 \mathrm{~nm}($ Figure $3 \mathrm{~A})$ and the following equation was obtained.

$$
\mathrm{Y}=0.0011 \mathrm{X}+0.0465
$$

Where $\mathrm{Y}$ is the absorbance value and $\mathrm{X}$ is the Doc concentration. The standard curve for the absorbance of Doc was linear over the range of $10-1000 \mu \mathrm{g} / \mathrm{ml}$ with a correlation coefficient of $\mathrm{R}^{2}=0.995$. Using the formula given in section 2.3.2 and equation (1), the encapsulation efficiency of Doc-nd (0.1\%DTX/2\%PFCE/2\%PEG-PDLA) was found to be 93.70\%. We have obtained very high values of encapsulation efficiency of Doc loaded in Doc-nd in comparison with Doc being loaded in other drug delivery systems like nanoparticles as published in other studies [14]. The perfluorocarbon in the core of the nanodroplets make these systems ultrasound-sensitive. Ultrasound is used to activate the release of docetaxel from these drug-delivery vehicles leading to increase in bioavailability. Other drug-delivery systems like nanoparticles do not have perfluorocarbon and rely only on natural degradation of the polymer for release of the drug. 
In vitro release profile

The release of Doc at $37^{\circ} \mathrm{C}$ from Doc-nd (0.1\%DTX/2\%PFCE/2\%PEG-PDLA) in PBS (pH 7.4) was evaluated by measuring UV absorbance at wavelength $227 \mathrm{~nm}$. The exact Doc concentration was calculated using absorbance values in equation 1 and these values were plotted with respect to time as showed in Figure 3B. The in vitro release mode of docetaxel from Doc-nd appeared to be three-staged. The profile revealed an initial burst of Doc release of $\sim 30 \%$ in the first hour. Subsequently, the release of the Doc was at a constant rate showing release up to $\sim 50 \%$ until $12 \mathrm{~h}$. After three days $\sim 85 \%$ of the Doc was released in the medium from the nanodroplets. Figure 3B features the controlled release pattern of Doc-nd, which might contribute to the prolonged circulation of these vehicles in vivo. The release profile would be helpful in our future in vivo studies in determining specific time points for the use of tumor-directed ultrasound to activate nanodroplets to release its entire drug loading and thus increasing tumor treatment efficacy while reducing the toxicity to the normal tissues.
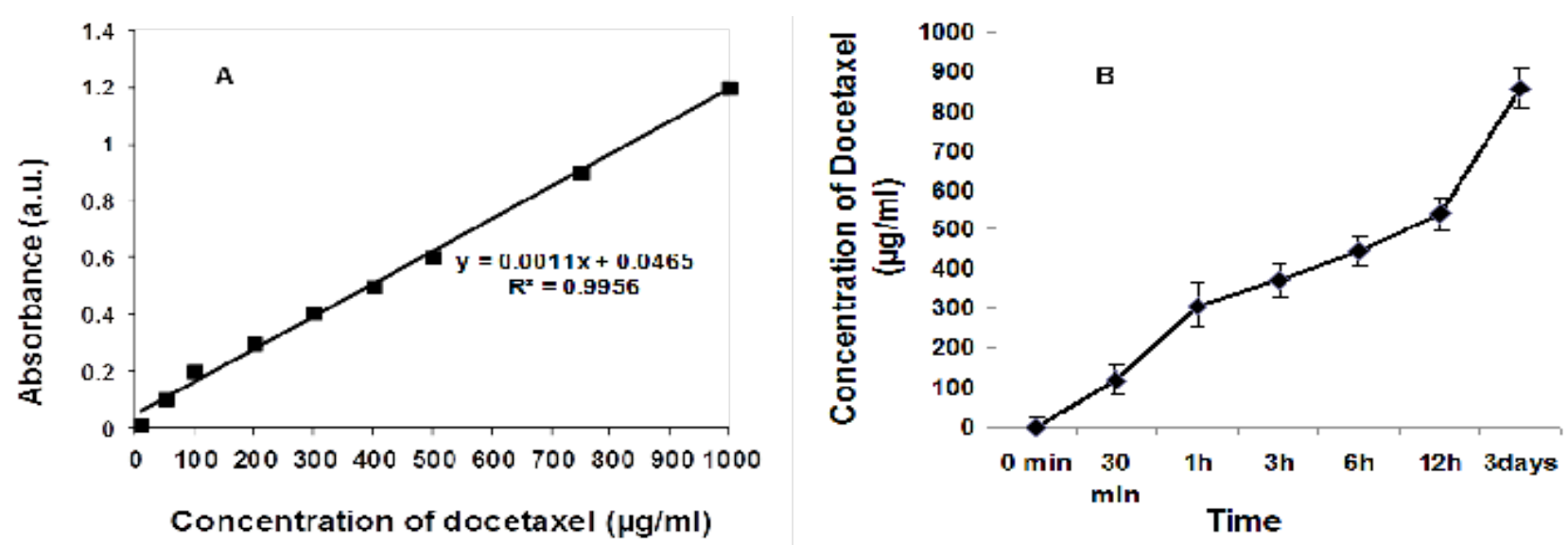

Figure 3: In vitro release kinetic study of docetaxel-loaded nanodroplets. (A) Standard absorbance calibration curve of different docetaxel concentration at $227 \mathrm{~nm}$. (B) Release of docetaxel in PBS from docetaxel-loaded nanodroplets at $37^{\circ} \mathrm{C}$.

In vitro cytotoxicity study

Cytotoxicity of Doc-nd (0.1\%DTX/2\%PFCE/2\%PEG-PDLA) against human prostate cancer LNCaP cells was measured using WST-1 assay (Figure 4). Cell viability was reduced to $~ 50 \%$ when incubated with Doc-nd for 2 min and decreased at a steady rate to $\sim 30 \%$ for up to one hour of incubation. During the $6 \mathrm{~h}$ of incubation, the cell viability was more or less stabilized at $\sim 30 \%$. This initial decrease of the cell viability indicated that docetaxel is being released steadily from Doc-nd with time. After $12 \mathrm{~h}$ and up to 3 days, the viability increased from $30 \%$ to $42 \%$. This increase in cell viability could be attributed to the growth of resistant and/ or survival cells. These results indicated a controlled release profile of Doc-nd when compared with cell viability for an equivalent concentration of free Doc. Only $20 \%$ cells survived when incubated with free Doc regardless of their incubation time (Figure 4).

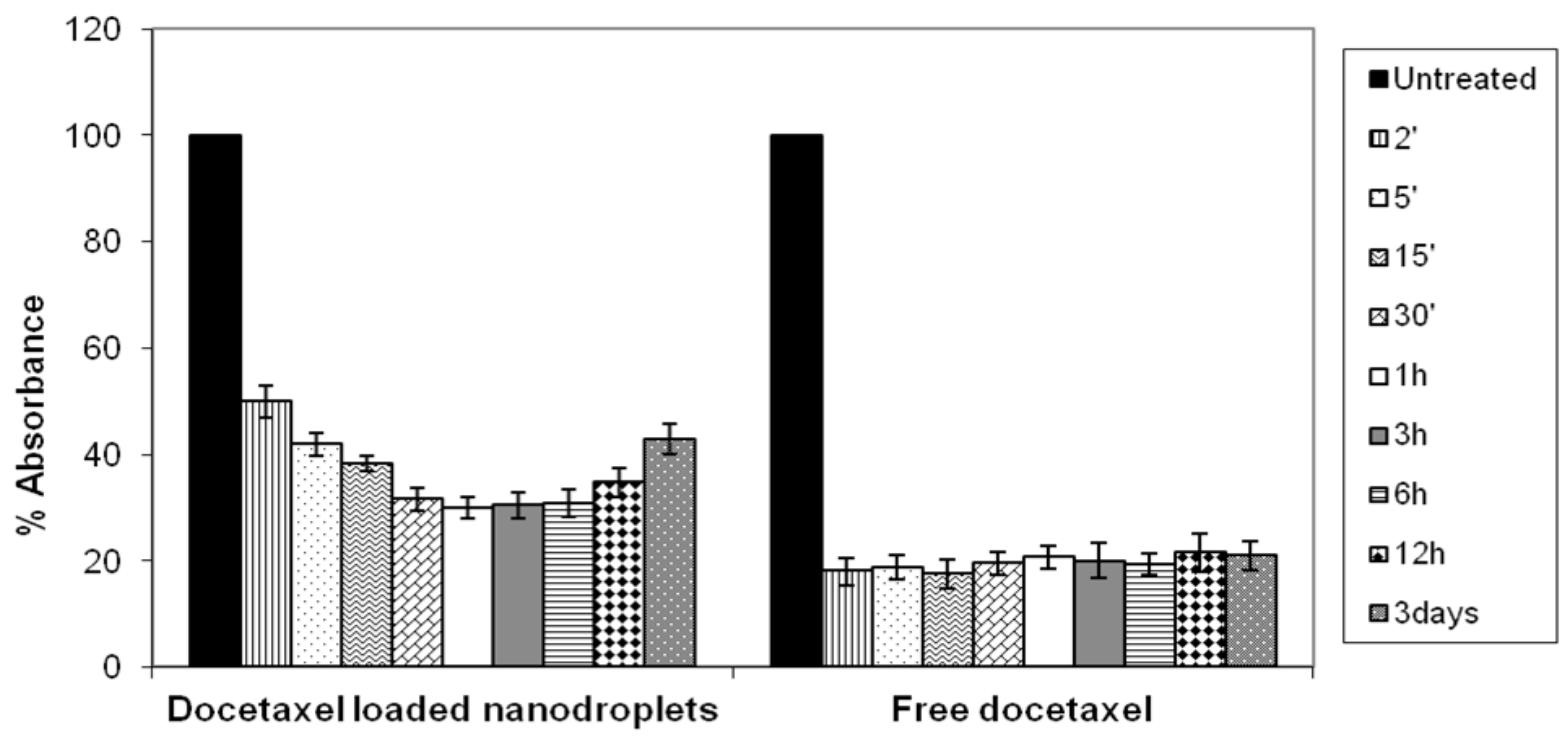

Figure 4: In vitro cytotoxic study of docetaxel-loaded nanodroplets and free docetaxel. The data (expressed as a percentage of untreated or control cells) is obtained after incubating LNCaP cells with docetaxel-loaded nanodroplets and free docetaxel for different time intervals and reading the assay using WST-1 
These results suggested that the concentration of free Doc used was high enough to induce $80 \%$ of cell killing with a probable loss of cellular reproduction capabilities as we did not observe an increase in viability upon a longer incubation time with free Doc when compared with Doc-nd. When the same concentration as of free Doc was encapsulated in nanodroplets (Doc-nd), cell viability increased and a sustained release cytotoxicity profile was observed. The controlled (time-dependent) cytotoxic release profile (Figure 4) also proves that Doc is encapsulated in the polymeric nanodroplets and is not readily available for cells to act upon as free Doc molecules of equivalent concentration. In an independent experiment, LNCaP cells were incubated with E-nd (2\%PFCE/2\%PEG-PDLA) and cell viability was assayed. The empty nanodroplets were found to be non-toxic to cells (data not shown) suggesting biocompatible properties of both PFCE and block co-polymer (PEG-PDLA) used in the synthesis of nanodroplets. Empty nanodroplets were also tested for their non-drug related toxicity in vivo on healthy male nude mice. We injected each of the three healthy mice with the empty nanodroplets twice a week, 15 times and monitored weight change over the course of 4 months. We found no significant weight change for these mice (unpublished data).

\section{Conclusion}

We have developed techniques for synthesis of the ultrasound-responsive Doc-loaded nanodroplets. We have characterized the novel formulations in terms of size and morphology. We have performed experiments for studying release kinetics and cell cytotoxicity studies. These studies are the first steps in realizing our long-term goal of increasing prostate cancer treatment efficacy by novel approach using Doc-loaded nanodroplets with MR-guided high intensity focused ultrasound exposures, while minimizing drug related toxicities. The efficacy of treating prostate cancer with this new approach under MR-guidance is currently being investigated in vivo using tumors grown orthotopically in the prostates of nude mice.

\section{Acknowledgement}

We would like to thank Dr Andrey Efimov for his technical assistance with bright field inverted microscope at bio-imaging facility at Fox Chase Cancer Center. This study was supported by DOD (PC073127), BC102806 and Varian Medical Systems.

\section{References}

1. Zhu Z, Zhang J, Liu Y, Chen M, Guo P, et al. (2014) Efficacy and toxicity of external-beam radiation therapy for localized prostate cancer: a network meta-analysis. Br J Cancer 110: 2396-404.

2. Bruce JY, Lang JM, Mc Neel DG, Liu G (2012) Current controversies in the management of biochemical failure in prostate cancer. Clin Adv Hematol Oncol 10: 716-22.

3. Management of localised prostate cancer: watchful waiting, surgery or radiation therapy, depending on the natural course, which is often relatively slow (2012) Prescrire Int 21: 242-8.

4. Petrylak DP, Tangen CM, Hussain MH, Lara PN, Jones JA, et al. (2004) Docetaxel and estramustine compared with mitoxantrone and prednisone for advanced refractory prostate cancer. N Engl J Med 351: 1513-20.

5. Tannock IF, de Wit R, Berry WR, Horti J, Pluzanska A, et al. (2004) Docetaxel plus prednisone or mitoxantrone plus prednisone for advanced prostate cancer. N Engl J Med 351: 1502-12.

6. Baker J, Ajani J, Scotte F, Winther D, Martin M, et al. (2009) Docetaxel-related side effects and their management. Eur J Oncol Nurs 13: 49-59.

7. Markman M (2003) Managing taxane toxicities. Support Care Cancer 11: 144-7.

8. Poi M, Berger M, Lutsberg M, Layman R, Shapiro CL, et al. (2013) Docetaxel-induced skin toxicities in breast cancer patients subsequent to paclitaxel shortage: a case series and literature review. Support Care Cancer 21:2679-86.

9. Lukka H, Waldron T, Chin J, Mayhew L, Warde P, et al. (2011) High-intensity focused ultrasound for prostate cancer: a systematic review. Clin Oncol 23: 117-27.

10. Warmuth M, Johansson T, Mad P (2010) Systematic review of the efficacy and safety of high-intensity focused ultrasound for the primary and salvage treatment of prostate cancer. Eur Urol 58: 803-15.

11. Gao Z, Kennedy AM, Christensen DA, Rapoport NY (2008) Drug-loaded nano/microbubbles for combining ultrasonography and targeted chemotherapy. Ultrasonics 48: 260-70.

12. Rapoport N, Kennedy AM, Shea JE, Scaife CL, Nam KH (2010) Ultrasonic nanotherapy of pancreatic cancer: lessons from ultrasound imaging. Mol Pharm 7: $22-31$.

13. Rapoport N, Nam KH, Gupta R, Gao Z, Mohan P, et al. (2011) Ultrasound-mediated tumor imaging and nanotherapy using drug loaded, block copolymer stabilized perfluorocarbon nanoemulsions. J Control Release 153: 4-15.

14. Zheng D, Li D, Lu X, Feng Z (2010) Enhanced antitumor efficiency of docetaxel-loaded nanoparticles in a human ovarian xenograft model with lower systemic toxicities by intratumoral delivery. Oncol Report 23: 717-24.

15. Iyer AK, Khaled G, Fang J, Maeda H (2006) Exploiting the enhanced permeability and retention effect for tumor targeting. Drug Discov Today 11: 812-8.

16. Hobbs SK, Monsky WL, Yuan F, Roberts WG, Griffith L, et al. (1998) Regulation of transport pathways in tumor vessels: role of tumor type and microenvironment. Proc Natl Acad Sci 95: 4607-12.

17. Campbell RB (2006) Tumor physiology and delivery of nanopharmaceuticals. Anticancer Agents Med. Chem 6: 503-12.

18. Wang J, Lu Z, Gao Y, Wientjes MG, Au JL (2011) Improving delivery and efficacy of nanomedicines in solid tumors: role of tumor priming. Nanomedicine 6: $1605-20$.

19. Kim SC, Kim DW, Shim YH, Bang JS, Oh HS, et al. (2001) In vivo evaluation of polymeric micellar paclitaxel formulation: toxicity and efficacy. J Control Release 72: 191-202. 
20. Chen L, Mu Z, Hachem P, Ma CM, Wallentine A, et al. (2010) MR-guided focused ultrasound: enhancement of intratumoral uptake of [ $\left.{ }^{3} \mathrm{H}\right]$-docetaxel in vivo. Phys Med Biol 55: 7399-410.

21. Mu Z, Ma CM, Chen X, Cvetkovic D, Pollack A, et al. (2012) MR-guided pulsed high intensity focused ultrasound enhancement of docetaxel combined with radiotherapy for prostate cancer treatment. Phys Med Biol 57: 535-45.

22. Hu Y, Ding Y, Ding D, Sun M, Zhang L, et al. (2007) Hollow chitosan/poly(acrylic acid) nanospheres as drug carriers. Biomacromolecules 8: $1069-76$.

23. Safavy A (2008) Recent developments in taxane drug delivery. Curr Drug Deliv 5: 42-54.

24. Zhang L, Yang M, Wang Q, Li Y, Guo R, et al. (2007) 10-Hydroxycamptothecin loaded nanoparticles: preparation and antitumor activity in mice. J Control Release 119: 153-62.

25. Rapoport N, Gao Z, Kennedy A (2007) Multifunctional nanoparticles for combining ultrasonic tumor imaging and targeted chemotherapy. J Natl Cancer Inst 99: 1095-106.

26. Davda J, Labhasetwar V (2002) Characterization of nanoparticle uptake by endothelial cells. Int J Pharm 233: 51-9.

27. Ling Y, Huang Y (2008) Preparation and release efficiency of Poly (lactic-co-glycolic) acid nanoparticles for drug loaded paclitaxel. IFMBE Proceedings 19: 514-7.

28. Musumeci T, Ventura CA, Giannone I, Ruozi B, Montenegro L, et al. (2006) PLA/PLGA nanoparticles for sustained release of docetaxel. Int J Pharm 325: 172-9.

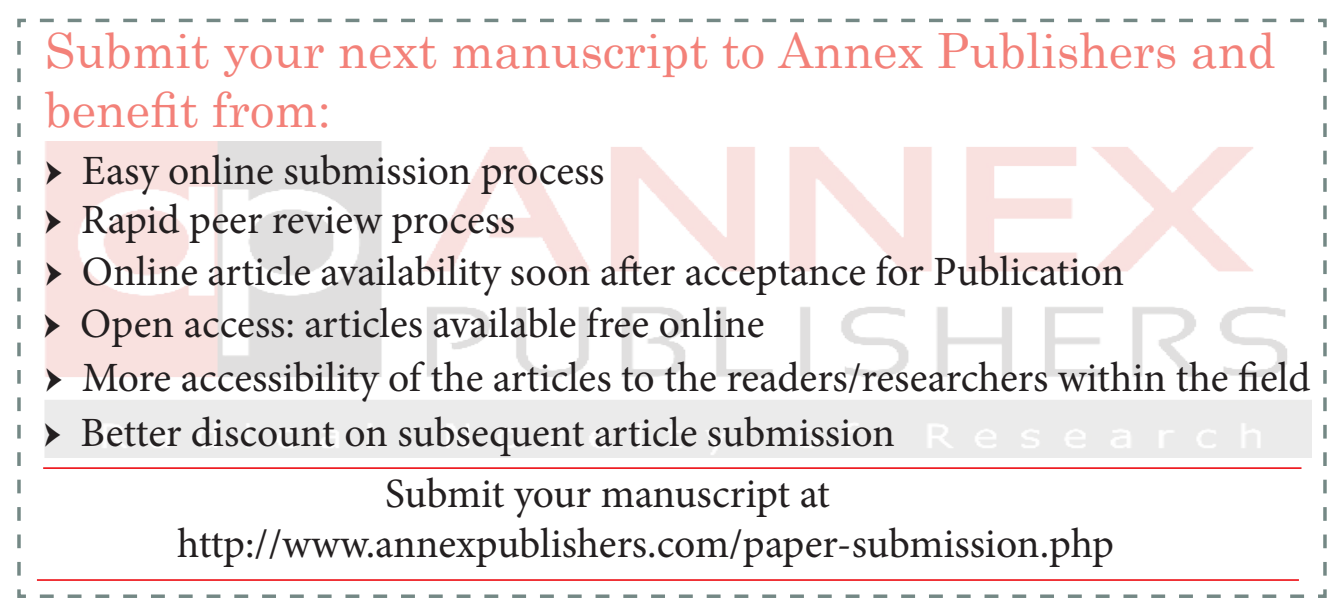

